\title{
Surface modifying substances that reduce apparent yeast cell hydrophobicity
}

\author{
LISA COLLING, MICHAEL ESSMANN, CARA HOLLMER, \& BRYAN LARSEN \\ Infectious Disease Research Laboratory, Des Moines University, Des Moines, IA, USA
}

(Received 28 fune 2004; accepted 10 November 2004)

\begin{abstract}
Objective. To determine whether several topical compounds and other chemical entities are able to diminish the surface hydrophobicity of yeast cells.

Method. Hydrophobicity of yeast cells was determined by binding styrene microspheres to the surface of untreated yeast or yeast pre-incubated with various substances with potential for cell surface modification. The degree of microsphere adherence to yeast cells was measured by flow cytometry.

Results. A significant reduction in cell surface hydrophobicity was observed when yeast was incubated in protein-containing media. Other compounds that effectively reduced microsphere binding were various formulations of K-Y ${ }^{\mathbb{R}}$ and heparin. Divalent cations $\left(\mathrm{Ca}^{++}, \mathrm{Mg}^{++}, \mathrm{Zn}^{++}, \mathrm{Cu}^{++}\right)$were also potent inhibitors of microsphere adherence. It was possible to remove substances contributing to microsphere binding by chemical extraction of the yeast. Yeast having reduced microsphere binding activity also showed diminished binding of concanavalin A.

Conclusions. Several commercially available compounds were able to block binding of styrene microspheres to yeast. Some of the binding activity appeared to be attributable to mannose-containing surface components. These findings have implications for formulating therapeutic products that might block yeast binding to tissues.
\end{abstract}

Keywords: Candida, virulence, adherence

\section{Introduction}

Our previous report [1] demonstrated the utility of blue-dyed styrene bead-binding to yeast cells detected by flow cytometry as an indicator of cell surface hydrophobicity. Interest in this cellular property derives from the putative role hydrophobicity plays in the adherence of fungal cells to mucosal tissues [2]. Determining the relative hydrophobicity might be useful in predicting the propensity of different strains to colonize mucosal tissues [3]. An additional observation we made was that different environmental conditions are able to alter the hydrophobicity of fungal organisms, suggesting that this characteristic might be subject to manipulation [1].

The goal of traditional therapies for mucocutaneous candidiasis has been focused on drug entities that interfere with the metabolism of yeast, resulting in death or at least stasis of growth. To our knowledge, the concept of blocking adherence to susceptible tissues has not been exploited in antifungal therapy. Most studies involving fungal adherence to epithelial tissues (human buccal cells, for example) have concentrated on identifying surface structures of yeast that are involved in binding to epithelial sites $[1,2]$. Since there is an extensive literature on the specific ligands possessed by yeast, the present report aims to determine whether readily available compounds, that are generally compatible with human tissues, have the ability to decrease the hydrophobicity of yeast. If successful, such compounds could be ideal tools for preventing or treating vaginal conditions.

\section{Methods}

Yeast strains

The present study included 12 clinical isolates described in detail in the earlier report ${ }^{1}$. These

Correspondence: Bryan Larsen, University Research, Des Moines University, 3200 Grand Avenue, Des Moines, IA 50312-4198, USA. Tel: +1 515271 1559. Fax: +1 515271 1644. E-mail: bryan.larsen@dmu.edu 
organisms were grown on Sabouraud's dextrose agar (SDA) for 24 hours at $25^{\circ} \mathrm{C}$ before use in microsphere-binding assays, unless otherwise indicated. Before the assay, a small amount of colonial growth was suspended in S-PBS sheath fluid (BectonDickinson, Franklin Lanes, NJ, USA), consisting of phosphate-buffered saline with $\mathrm{KCl}$ and disodium ethylene diamine tetra-acetic acid (EDTA). The optical density of the yeast suspension was adjusted to $\mathrm{A}_{600}=1.0 \pm 0.1$

\section{Microsphere-binding assay}

Deep blue-dyed styrene microspheres $(0.83 \mu \mathrm{m})$ were obtained from Sigma-Aldrich (St Louis, MO, USA); microspheres comprising $10 \%$ solids were supplied in $1 \mathrm{ml}$ volumes. Subsequently, an additional $3 \mathrm{ml}$ of S-PBS was added to the suspension. At the time of binding assay, $10 \mu \mathrm{l}$ of the yeast suspension was diluted into $1 \mathrm{ml}$ of S-PBS, and $10 \mu \mathrm{l}$ of the diluted microsphere suspension was added. The microsphere/yeast mixture placed on a rotator at $14 \mathrm{rpm}$ for 30 minutes at room temperature, followed by flow cytometry.

\section{Flow cytometry}

This measured forward scatter (FSC), side scatter (SSC) and Fl3 channel fluorescence (Fl3-h). Microspheres alone and yeast alone were analyzed to establish parameters that allowed analysis of yeast without appreciable interference by unbound microspheres (data not shown). Flow cytometry data were presented as percentage of particles showing fluorescence above a preset fluorescence threshold (\%M1) and the mean $\mathrm{Fl3}-\mathrm{h}$ associated with yeast (MCF-Fl3-h); 10,000 events were counted for each sample, unless the particles in the sample were in low abundance, when 5,000 events were counted.

\section{Test substances}

Several compounds were used to pretreat yeast for 30 minutes at room temperature before microsphere-binding assays, establishing their efficacy as cell surface hydrophobicity modifiers. Reagent grade $\mathrm{CaCl}_{2}, \mathrm{MgSO}_{4}, \mathrm{ZnSO}_{4}$ and $\mathrm{CuSO}_{4}$ were obtained from Fisher Scientific (Pittsburg, PA, USA) and dissolved in water, or in S-PBS in the case of $\mathrm{MgSO}_{4}$. Heparin sulfate was preformulated to $100 \mathrm{u} / \mathrm{ml}$ by Abbot Laboratories (North Chicago, IL, USA) and $\mathrm{K}-\mathrm{Y}^{\circledR}$ products $\left(\mathrm{K}-\mathrm{Y}^{\circledR}\right.$ gel, $\mathrm{K}-\mathrm{Y}^{\circledR}$ liquid, $\mathrm{K}-\mathrm{Y}^{\circledR}$ ultra and $\mathrm{K}-\mathrm{Y}^{\mathbb{R}}$ warming) were provided courtesy of the manufacturer, Advanced Care Products (Raritan, NJ, USA).

\section{Cell surface stripping and concanavalin A staining}

The method of Poulain et al. [4] was used to treat yeast cells. A solution of sodium dodecyl sulfate (4\%), $50 \mathrm{mM}$ EDTA and 5\% 2-mercaptoethanol was mixed with yeast cells vigorously for 30 minutes. Yeasts were harvested by centrifugation at 10,000 rcf for 15 minutes and then resuspended in S-PBS. Resuspended cells were reacted with microspheres or with fluoresceinated concanavalin A (Sigma-Aldrich, St. Louis, MO, USA) used as a 1:100 dilution of a $10 \%$ stock solution. Concanavalin A-treated cells were analyzed in fluorescence channel 1 (Fl1-h).

\section{Statistics}

All data collected represented continuous variables (\%M1 and MCF-Fl3-h) and were evaluated by paired comparisons using Student's $t$-test; $p<0.05$ was considered significant. Because reduction in fluorescence was being sought in this study, percentage reduction in \% 11 compared with control and percentage reduction in MCF-Fl3-h compared with control were also calculated, i.e., 1-(experimental \% $\mathrm{M} 1 /$ control \% M1) x 100, or 1-(experimental MCFFl3-h/control MCF-Fl3-h) x 100.

\section{Results}

Growth of Candia albicans under different conditions was previously shown to affect surface hydrophobicity [1]. As a result, we also compared hydrophobicity after growth in yeast nitrogen broth (YNB) or in Sabouraud's dextrose broth (SAB). As shown in Figure 1, the latter medium produced yeast which generally bound microspheres less effectively than cells grown in yeast nitrogen broth. Reduction in percentage M1 occurred with 11 of 12 strains, and reduction in $\mathrm{MCF}-\mathrm{Fl} 3-\mathrm{h}$ occurred in half of test strains.

Although a key difference between YNB and SAB media was the presence or absence of protein, it was not clear whether the protein in $\mathrm{SAB}$ medium was altering the expression of binding sites on the SABgrown yeast or if it was interacting directly with the yeast to mask the microsphere-binding sites. To answer this question, we pre-incubated each of the Candida albicans strains with $1 \%$ bovine serum albumin (BSA) for 30 minutes before microspherebinding assay. Figure 2 shows the results of this study, in which fluorescence due to microsphere binding was decreased by previous exposure to RSA for all 12 strains tested. The reduction in percentage $M 1$ compared with controls without BSA ranged from $48 \%$ to $56 \%$, with BSA effects reaching statistical significance for both \%M1 and MCF-Fl3-h.

Having found that BSA exposure was able to alter hydrophobicity of yeast surfaces, we began seeking 

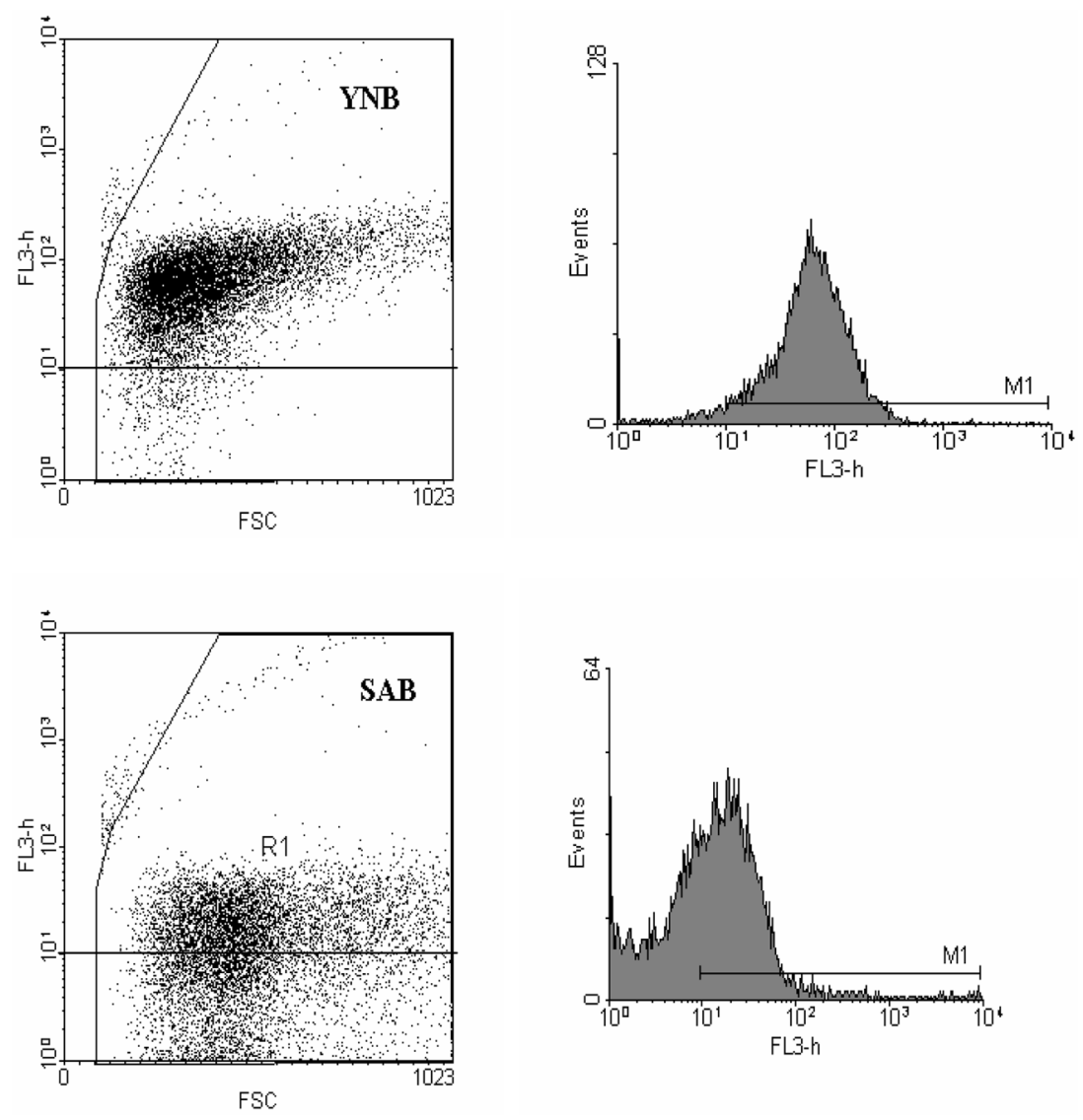

Figure 1. Effect of protein-containing medium on binding of microspheres to yeast strain 387. Yeasts were grown over night at $25^{\circ} \mathrm{C}$ in either yeast nitrogen broth (which contains no protein) or Sabourand's dextrose broth (SAB) (which contains 0.5\% peptone). Yeasts which have bound styrene microspheres appear in region 1 (R1) of the plot (left panels). Histogram plots (right panels) show the relative fluorescence (FL3-h) of the yeast, indicating decreased microsphere binding with protein-containing medium. FSC, forward scatter; YNB, yeast nitrogen broth.

other materials that could alter microsphere binding. We examined the role of divalent cations $\mathrm{Ca}^{++}$and $\mathrm{Mg}^{++}$as shown in Figure 3. $\mathrm{CaCl}_{2}$ was dissolved in sterile water, as it forms insoluble precipitates with $\mathrm{S}$ PBS. Dilutions of $\mathrm{Ca}^{++}$and $\mathrm{Mg}^{++}$were used to pretreat each of the 12 strains of yeast before assay. Both cations effectively reduced microsphere binding in a dose-responsive manner. We also determined if transition metal salts $\mathrm{ZnSO}_{4}$ or $\mathrm{CuSO}_{4}$ reduced binding and a similar effect was observed; concentrations above $50 \mathrm{mM}$ of $\mathrm{Ca}^{++}, \mathrm{Mg}^{++}$and $\mathrm{Zn}^{++}$as well as concentrations above $1 \mathrm{mM}$ of $\mathrm{Cu}^{++}$significantly decreased microsphere binding.

We next determined if heparin sulfate reduced adherence. As shown in Figure 4, both fluorescence parameters generally were reduced by exposure to heparin sulfate, although not as profoundly as for BSA or divalent cations. Reductions for percentage M1 proved statistically significant by paired $t$-test, although MCF-Fl3-h was not significantly lowered in the presence of heparin. We did discover, however, that certain strains were less affected by heparin than others. For example percentage MI ranged from $1 \%$ reduction in 1 strain to a maximum of $44 \%$ reduction in the other 11 yeast strains. Similarly, 2 strains showed an increase in MCF-Fl3$\mathrm{h}$ after heparin treatment, but the remaining strains showed reductions of up to $41 \%$.

The possibility that already-marketed intravaginal products might inhibit binding was evaluated by pre-incubating several $\mathrm{K}-\mathrm{Y}^{\circledR}$ products with each of the 12 clinical yeast isolates available. Because of the viscosity of $\mathrm{K}-\mathrm{Y}^{\circledR}$ jelly, it was diluted 1:10 (weight/volume) in S-PBS before addition of yeast cells. The various liquid forms of $\mathrm{K}-\mathrm{Y}^{\circledR}$ (ultra $^{\mathrm{TM}}$, liquid $^{\mathrm{TM}}$ and warming ${ }^{\mathrm{TM}}$ ) were used at full strength. After a 30-minute incubation, yeasts were recovered, resuspended in S-PBS and reacted with styrene microspheres. The relative effects on binding are presented in Figure 5. Despite its dilution, the original $\mathrm{K}-\mathrm{Y}^{\circledR}$ jelly form was very effective in decreasing fluorescence due to microsphere binding and ultra ${ }^{\mathrm{TM}}$ appeared the most effective. Binding was decreased by ultra ${ }^{\mathrm{TM}}$ in all strains tested: decreases in percentage M1 ranged from $31 \%$ to $64 \%$, and comparable decreases in MCF-Fl3-h ranged from $46 \%$ to $86 \%$. Average percentage M1 and average MCF-Fl3-h for the 12 


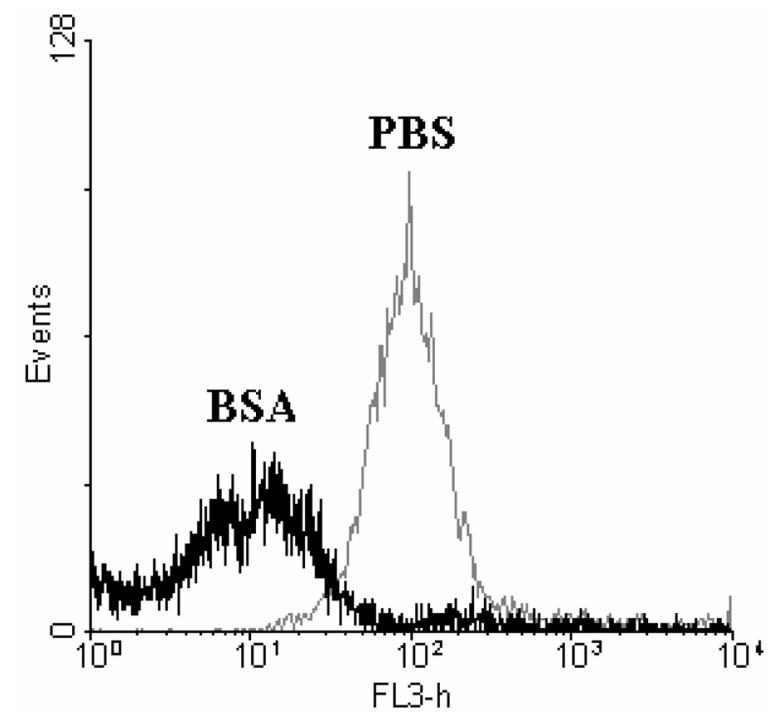

Figure 2. Incubation with $1 \%$ bovine serum albumin (BSA) decreased styrene microsphere binding. Yeast strain 132 was suspended in S-PBS or S-PBS with 1\% BSA for 30 minutes before addition of blue-dyed styrene microspheres. The flow cytometer was gated to exclude unbound microspheres, and the histograms for the two conditions overlaid. BSA-treated yeast showed decreased fluorescence, $(\mathrm{Fl} 3-\mathrm{h})$ indicating reduced bead binding.

strains were statistically significant for all $\mathrm{K}-\mathrm{Y}^{\circledR}$ formulations.

We next determined whether we could decrease bead adhesion by removing cell surface components from yeast cells before binding assays. We used a cocktail of EDTA, 2-mercaptoethanol and SDS, described in the methods with results shown in Figure 6. As illustrated, binding determined by percentage $\mathrm{M} 1$ and MCF-Fl3-h was decreased, but only to a limited extent. This ranged from $0 \%$ to $62 \%$ reduction in percentage M1 and from $25 \%$ to $78 \%$ reduction in MCF-Fl3-h. Because yeast-totissue adherence, and in particular tissue adherence based on hydrophobicity, has been associated with mannose-substituted proteins, we evaluated relative fluorescence resulting from binding concanavalin A to mannose groups on control cells and yeast cells extracted with SDS-EDTA 2-mercaptoethanol. As demonstrated in Figure 6, at least a portion of the surface material responsible for microsphere adherence comprised mannose-containing substances, such as concanavalin A: binding was decreased in cells extracted with SDS/2-mercaptoethanol/EDTA.

\section{Discussion}

The development of potent antifungal drugs may have diminished the urgency for developing novel antifungal topical products, but the continuing perplexing problem of vaginal candidiasis, particularly in its recurrent form, suggests that there may yet be new opportunities for attacking virulence factors in pathogenic fungi for the purpose of enhancing therapeutic options. This study serves as a proof of concept for an approach that diminishes a virulence factor in yeast, namely cell surface hydrophobicity.

The idea of attacking microbial diseases by directly interfering with a virulence factor has been applied to organisms with one overwhelming virulence property, such at tetanus toxin in the case of Clostridium tetani for which antibody specific for tetanus toxin is able to effectively prevent disease. However, the role of virulence factors in opportunistic organisms is more complex than in frank pathogens. As noted previously, disease caused by opportunists may depend on synergy between a set of several virulence attributes [5]. For example, in the case of Candida albicans, secreted proteinases [6], dimorphic growth [7], complement receptor-like ligands [8], enzymes that create covalent links with host cells [9], in addition to cell surface hydrophobicity, are reputed to contribute to virulence. If simultaneous expression of multiple virulence attributes is required for Candida to cause symptomatic infection, then disruption of one of these factors could have an important role in diminishing the ability of the organism to maintain symptomatic infection. Thus, whereas surface hydrophobicity may not represent all of the cell's virulence capability, development of ways to reduce its impact may be beneficial in controlling yeast infections.

The finding that proteins, divalent cations and some already-marketed intravaginal products may alter apparent yeast hydrophobicity raises the possibility of using such products to decrease yeast adherence to epithelia. Although it may not be practical to use these compounds alone, particularly when standard practice relies on potent specific antifungals, such adherence-defeating products might be appropriate adjuncts to specific antifungal drugs, or attractive vehicles for delivery of therapeutic products. These uses would require additional studies, including demonstration of reduced yeast adherence to epithelial cells. Such studies would also need to be accompanied by animal and clinical investigation.

Recognizing that observations on yeast binding to epithelial cells would lend additional clinical relevance to our observations, we performed preliminary studies to see whether flow cytometric methods could be applied to epithelial cells harvested from buccal mucosa. We analyzed the fluorescence pattern of freshly harvested cells and found a very high level of autofluorescence in these cells. We concluded that it was unlikely that we would be able to distinguish fluorescent labeled yeast from the background autofluorescence of these cells. As a result, we decided that flow cytometric studies of yeast binding to epithelial cells would have to await the discovery of an alternative source of human 

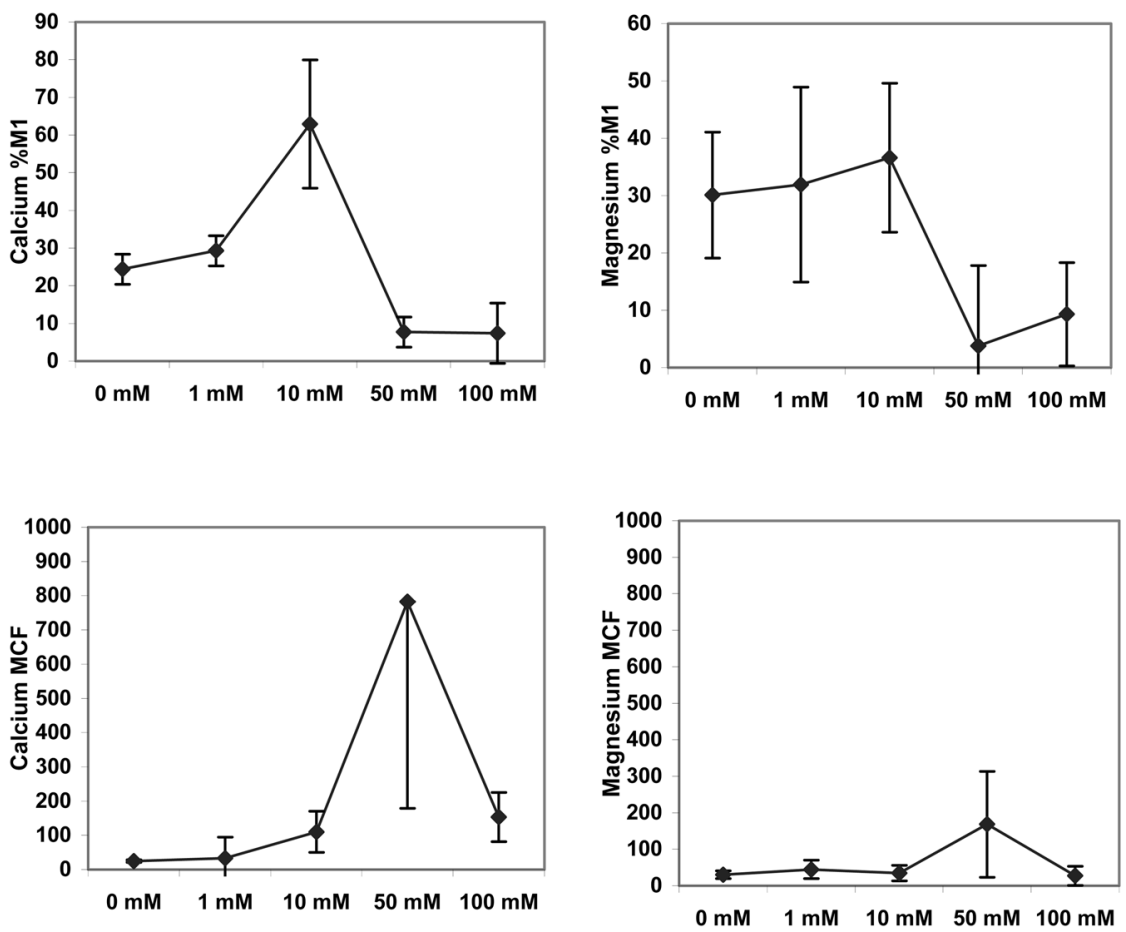

Figure 3. Effect of divalent cations on microsphere binding. Bead-binding assays were performed in sterile water with varying concentrations of $\mathrm{Ca}^{++}(\mathrm{CaCl} 2)$ or $\mathrm{Mg}^{++}(\mathrm{MgSO} 4)$. The percentage of yeast cells showing fluorescence $(\% \mathrm{M} 1)$ is presented in the upper panels. Data are for 12 clinical yeast isolates, and standard deviation is shown by error bars. The relative fluorescence (MCF-Fl3-h) is shown in lower panels. Both parameters indicate that divalent cations reduced microsphere binding compared with controls.
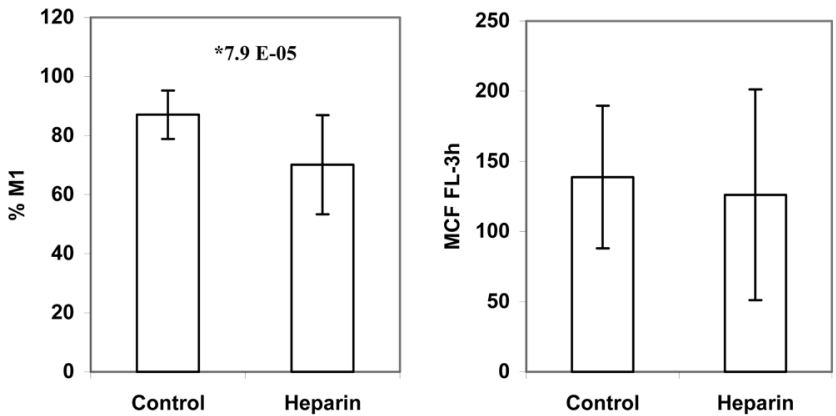

Figure 4. Heparin sulfate effects on yeast surface hydrophobicity. Twelve yeast strains were pre-incubated at $25^{\circ} \mathrm{C}$ with $100 \mu \mathrm{l}$ heparin sulfate or with S-PBS, before bead-binding assay. The percentage M1 (average of 12 strains) was reduced, and many yeast strains showed reduced relative fluorescence (MCF-F13-h), although on average the parameter was not significant. Asterisk indicates $p<0.05$.

epithelial cells that would not pose the problem of significant background fluorescence.

Despite the fact that we were not at this time able to investigate yeast binding to epithelial cells, the present study provided an interesting way of probing the surface of yeast and suggested that the interaction of the microspheres with yeast was a complex system, based on more factors than a single, specific and easily characterized ligand. Indeed, the fact that exposure to divalent cations profoundly reduced microsphere binding, but the polyanion, heparin sulfate also reduced binding (although less consistently and much less effectively than divalent metals) is somewhat difficult to explain in terms of a single binding mechanism for adherence of microspheres to yeast. Vardar-Unlu and colleagues $(10)$ found $\mathrm{Ca}++$ and $\mathrm{Mg}++$ were not needed for fucose binding to yeast, but Alonso et al. [11] found these same cations promoted yeast binding to collagen. Clearly, there are differences among various substances that interact with yeast surfaces and, whereas divalent cations may enhance some binding interactions, they clearly do not do so in all cases. In the case of hydrophobicity as measured by microsphere binding, the addition of metallic cations diminished binding activity. It should be noted that, because the experiments with divalent cations used both monovalent and divalent counterions in the experiments 

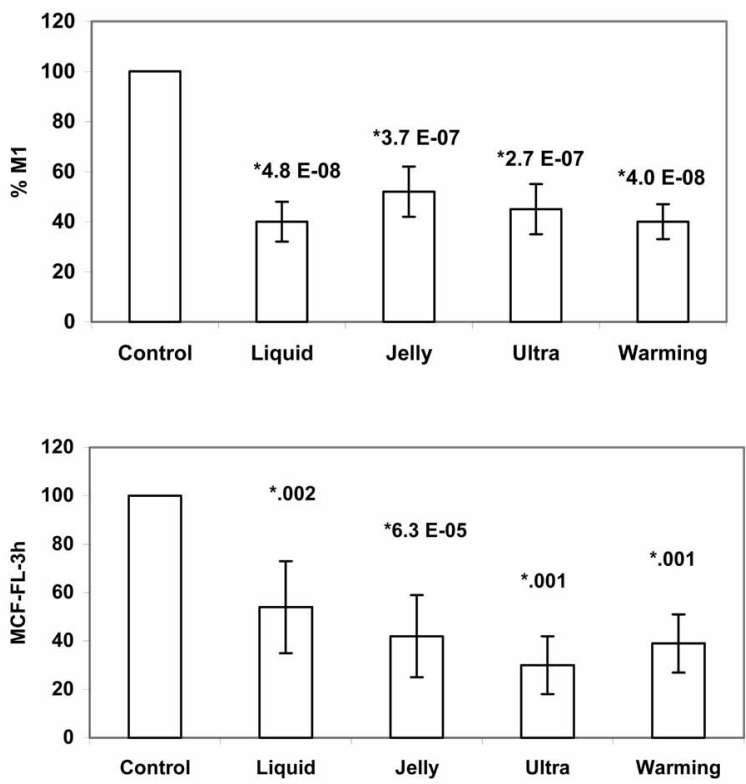

Figure 5. Effect of marketed intravaginal products on relative yeast cell hydrophobicity. Liquid formulations of $\mathrm{K}-\mathrm{Y}^{\circledR}$ personal lubricant were used to suspend yeast before bead-binding assay. Standard K-Y ${ }^{\circledR}$ jelly was diluted 1:10 before suspending yeast, because of its much greater viscosity. Following a 30-minute exposure to the $\mathrm{K}-\mathrm{Y}^{\circledR}$ products, yeasts were harvested by centrifugation at 10,000 $\mathrm{rcf}$ for 15 minutes, the supernatant was removed, the pelleted yeasts were resuspended in S-PBS and styrene microspheres were added. All formulations reduced bead binding, $\mathrm{K}-\mathrm{Y}^{\circledR}$ ultra having the greatest effect. Asterisk indicates $p<0.05$.

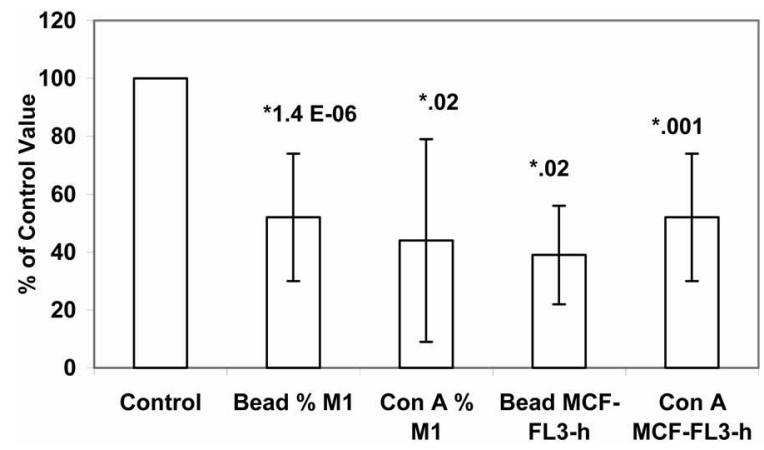

Figure 6. Extraction of cell surface proteins reduces yeast hydrophobicity. Cellular surface proteins were stripped from 12 strains of yeast by treating them with 2ME-SDS-EDTA (see Methods). Both bead-binding parameters were decreased by the stripping procedure, as was binding of fluoresceinated concanavalin $\mathrm{A}$.

(sulfate for $\mathrm{Mg}$ and chloride for $\mathrm{Ca}$ ), we concluded that the observed decrease in inhibition of binding activity was due to the cation.

In view of the diversity of compounds that appeared to decrease cell hydrophobicity, it is unlikely that their individual activities were due to a single mechanism. This is well illustrated by the different personal lubricant products tested. Each of these products contain unique compositions, including the components that lend viscosity to the products (according to the manufacturer's labeling). A similar decrease in binding was seen with cells stripped of selected surface proteins by the method of Poulain et al. [4], but clearly the stripped cells had diminished levels of mannosesubstituted proteins on their surfaces as evidenced by the concomitant loss of concanavalin A staining. It is almost certain that microsphere binding will not be shown to rely on a single species of surface molecule on the yeast.

The stripping of various surface molecules from yeast has been reported previously [4], and we based our chemical extraction of yeast on this work. Mannose proteins which are detected by interaction with concanavalin A have been implicated previously as contributing to hydrophobicity of yeast. Further characterization of materials removed from cells, rendering them less adherent, could provide a moderately specific adherence-blocking agent. Despite the apparent complexity of specific cellular components that contribute to cell hydrophobicity, the inhibitors seem remarkably consistent in their ability to alter microsphere binding. By using multiple clinical isolates in this study we demonstrated that, whereas there is some quantitative variation among strains, hydrophobicity-reducing measures affected most strains, suggesting that such intervention might prove to be generally applicable in clinical practice. The next obvious steps in this process will be to design appropriate clinical trials to extend the concepts developed through this study.

\section{References}

1. Colling L, Carter RN, Essmann M, Larsen B. Evaluation of relative yeast cell surface hydrophobicity measured by flow cytometry. Infect Dis Obstet Gynecol 2005;13(1):43-48.

2. Hazan KC. Participation of yeast cell surface hydrophobicity in adherence of Candida albicans to human epithelial cells. Infect Immun 1989;57:1894-1900.

3. Ener B, Douglas LJ. Correlation between cell-surface hydrophobicity of Candida albicans and adhesion to buccal epithelial cells. FEMS Microbiol Lett 1992;78:37-42.

4. Poulain D, Slomianny C, Jouault T, Gomez JM, Trinel PA. Contribution of phospholipomannan to the surface expression of beta-1,2-oligomannosides in Candida albicans and its presence in cell wall extracts. Infect Immun 2002;70(8): 4323-4328.

5. Cutler JE. Putative virulence factors of Candida albicans. Annu Rev Microbiol 1991;45:187-218.

6. Naglik JR, Challacombe SJ, Hube B. Candida albicans secreted aspartyl proteinases in virulence and pathogenesis. Microbiol Mol Biol Rev 2003;67(3):400-428.

7. Liu H, Kohler J, Fink GR. Suppression of Hyphal formation in Candida albicans by mutation of a STE12 homolog. Science 1994;266:1723-1725. 
8. Gilmore BJ, Retsinas EM, Lorenz JS, Hostetter MK. An iC3b receptor on Candida albicans: structure, function and correlates for pathogenicity. J Infect Dis 1988;157:38-46.

9. Staab JF, Bradway SD, Fidel PL, Sundstrom P. Adhesive and mammalian transglutaminase substrate properties of Candida albicans Hwp1. Science 1999;283:1535-1538.

10. Vardar-Unlu G, McSharry C, Douglas LJ. Fucose specific adhesive on germ tubes of Candida albicans. FEMS Immunol Med Microbiol 1998;20:55-67.
11. Alonso R, Llopis I, Flores C, Murgui A, Timoneda J. Different adhesions for type IV collagen on Candida albicans: identification of a lectin-like adhesion recognizing the 7s (IV) domain. Microbiology 2001;147:1971-1981.

12. Rodier MH, Imbert C, Kauffmann-Lacroid C, Daniault G, Jaquemin J. Immunoglobulins G could prevent adherence of Candida albicans to polystyrene and extracellular matrix components. J Med Microbiol 2003;52:373-377. 


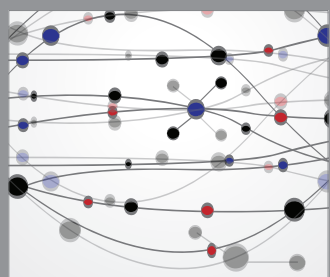

The Scientific World Journal
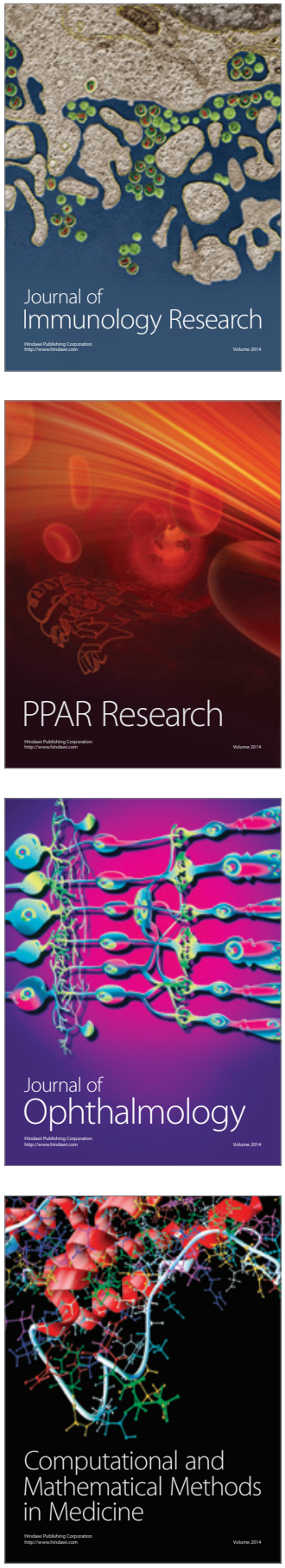

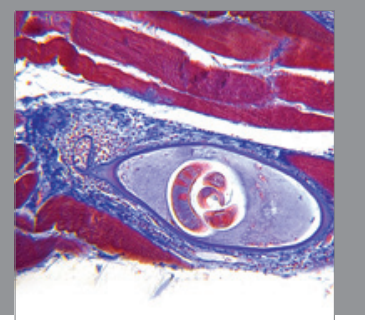

Gastroenterology

Research and Practice
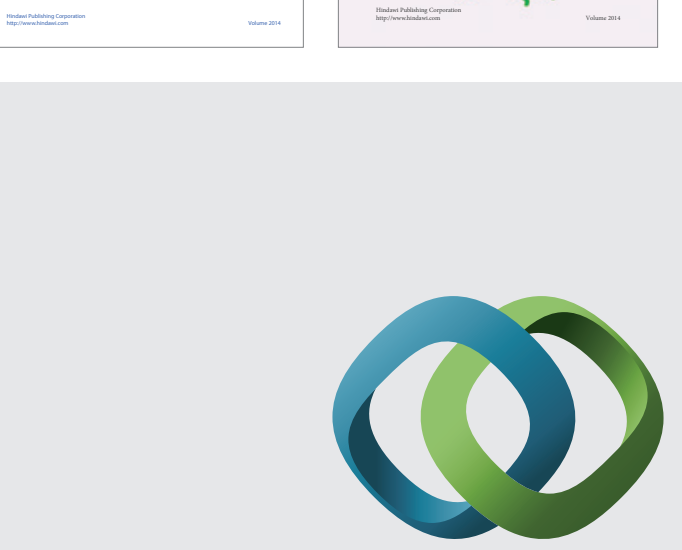

\section{Hindawi}

Submit your manuscripts at

http://www.hindawi.com
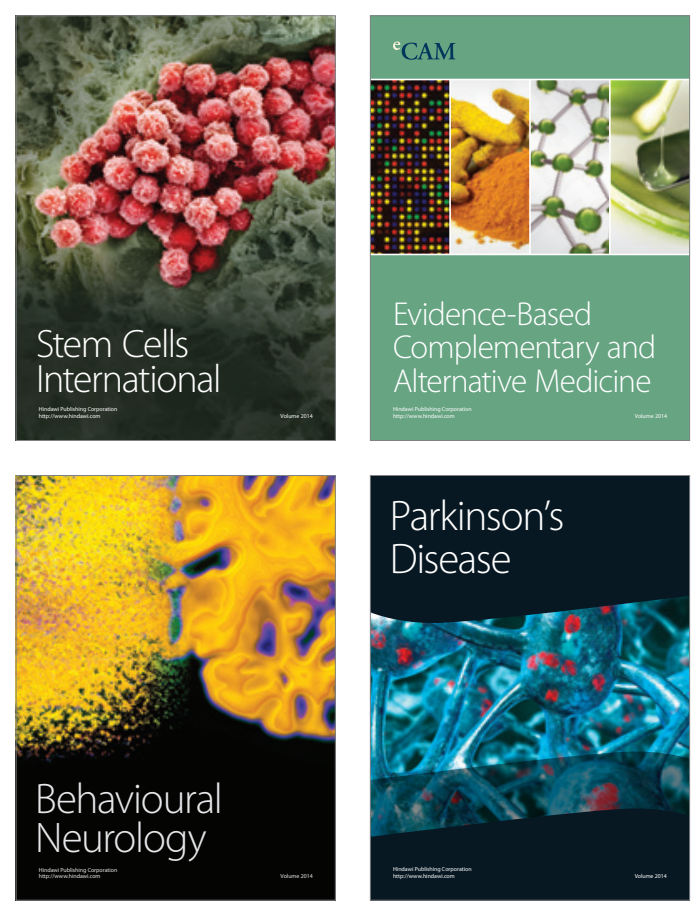

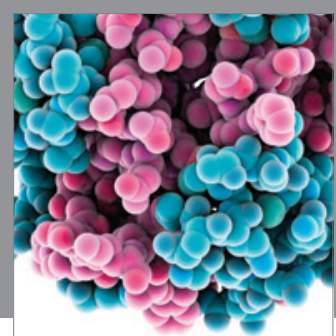

Journal of
Diabetes Research

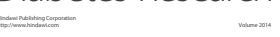

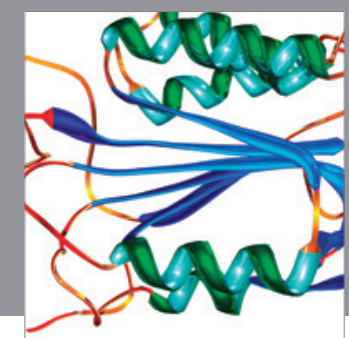

Disease Markers
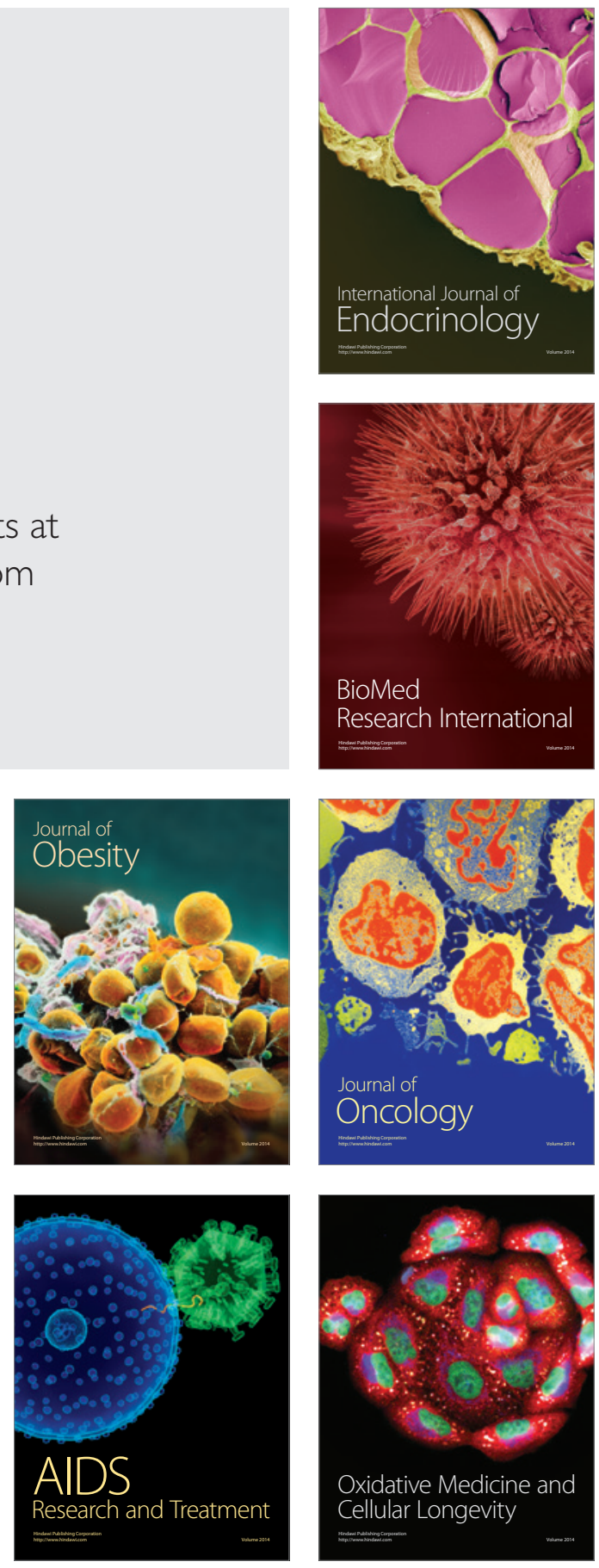